\title{
Erratum to: Effective temperature of active fluids and sheared soft glassy materials ${ }^{\star}$
}

\author{
Saroj Kumar Nandi and N.S. Gov ${ }^{\mathrm{a}}$ \\ Department of Chemical and Biological Physics, The Weizmann Institute of Science, Rehovot 76100, Israel
}

Original article: Eur. Phys. J. E (2018) 41: 117, https://doi.org/10.1140/epje/i2018-11731-7

Received: 16 January 2019

Published online: 14 February 2019

(c) EDP Sciences / Società Italiana di Fisica / Springer-Verlag GmbH Germany, part of Springer Nature, 2019

It was pointed out to us by our collaborators Golan Bel and Dan Wexler that the expression for the overdamped limit of the kinetic energy of the trapped particle, that we have used $[1,2]$, was in error. In this erratum we provide the correct expressions which Bel and Wexler have derived, while noting that qualitatively all the results and conclusions of the original paper remain unchanged [2].

The correct expressions for the kinetic and potential energies, not restricted to the overdamped limit, are

$$
T_{\mathrm{kin}}^{a}=\frac{1}{2} m_{\mathrm{tr}}\left\langle v^{2}\right\rangle_{a}=\frac{m_{\mathrm{tr}} p_{\mathrm{on}} N_{m} f_{0}^{2}}{4 \lambda^{2}\left(1+\frac{m_{\mathrm{tr}}}{\lambda \tau_{p}}+\frac{k \tau_{p}}{\lambda}\right)}
$$

and

$$
T_{\mathrm{pot}}^{a}=\frac{1}{2} k\left\langle x^{2}\right\rangle_{a}=\frac{m_{\mathrm{tr}} p_{\text {on }} N_{m} f_{0}^{2}\left(1+\lambda \tau_{p} / m_{\mathrm{tr}}\right)}{4 \lambda^{2}\left(1+\frac{m_{\mathrm{tr}}}{\lambda \tau_{p}}+\frac{k \tau_{p}}{\lambda}\right)} .
$$

Comparing to the simulation data $[3-5]$ we therefore fit the kinetic energy of the tracer particle to a function of the form

$$
T_{\mathrm{kin}}^{a}=m_{\mathrm{tr}} \frac{T_{\mathrm{kin}, 0}^{a}}{\left(A+m_{\mathrm{tr}}\right)},
$$

where $A=\lambda \tau_{p}\left(1+k \tau_{p} / \lambda\right)$. We find that for both of the data sets of the active fluids [3,4] we can get reasonably good agreement with eq. (3) (fig. 1(a), (b)), using the fit parameters $A=40,200$, respectively.

Fitting eq. (3) to the simulations of sheared soft glassy material (SGM) [5], the agreement is less good (fig. 1(c)). As we noted in [2], in this system there seems to be a scaling of $T_{\text {kin }}^{a} \propto m_{\mathrm{tr}}^{1 / 2}$, which is not accounted for by our simple model.

Regarding the question of the relation between the value of the long-time effective temperature extracted from the generalized Fluctuation-Dissipation Theorem (FDT), and the kinetic energy of the tracer, we now have the following two ratios predicted by our model:

$$
\frac{T_{\mathrm{FDT}, 0}}{T_{\mathrm{kin}}}=1+\frac{\lambda \tau_{p}}{m_{\mathrm{tr}}}+\frac{\tau_{p}^{2} k}{m_{\mathrm{tr}}} \quad \text { and } \quad \frac{T_{\mathrm{pot}}}{T_{\mathrm{kin}}}=1+\frac{\lambda \tau_{p}}{m_{\mathrm{tr}}} .
$$

Note that both of these ratios approach one in the limit of $\tau_{p} \rightarrow 0$, where there is no effect of activity and equipartition is recovered. Both ratios also approach one in the limit of $m_{\mathrm{tr}} \rightarrow \infty$.

The predictions made at the end of the paper [2] regarding the effects of changing the tracer particle's radius need to be slightly revised: In $3 \mathrm{D}$, for viscous friction $(\mu=1)$, we therefore get from eqs. (1), (3) that $T_{\text {kin }}^{a} \propto$ $R /\left(1+A_{1} R^{2}+A_{2} / R\right)$, which increases with increasing tracer size for small $R$, but decreases for large $R$.

All other predictions made in the paper remain correct.

\footnotetext{
* Contribution to the Topical Issue "Flowing Matter, Problems and Applications" edited by Federico Toschi, Ignacio Pagonabarraga Mora, Nuno Araujo, Marcello Sega.

a e-mail: nir.gov@weizmann.ac.il (corresponding author)
} 

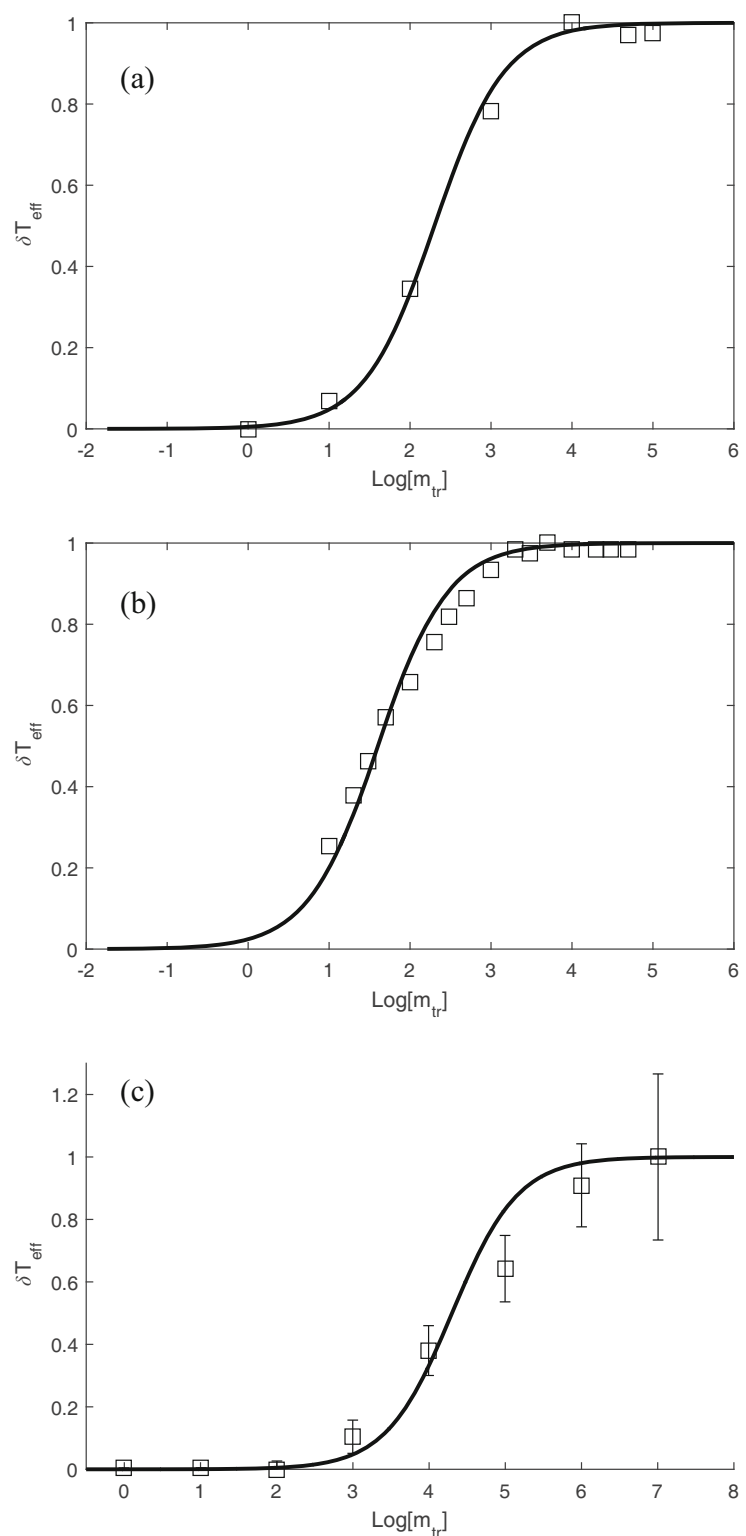

Fig. 1. Comparison between the normalized increase in the mean kinetic energy of the tracer particle: $\delta T_{\text {eff }}=\left(T_{\text {eff }}-T\right) /\left(T_{\text {eff }}-\right.$ $T)\left.\right|_{\left(m_{\mathrm{tr}} \rightarrow \infty\right)}$, as a function of the tracer particle's mass $m_{\mathrm{tr}}$ (where $T_{\text {eff }}=T_{\mathrm{kin}}^{a}+T$ ). The fits using Eq.3 are given by the thick solid line, using $A=200,40,2 \times 10^{4}$, for (a), (b), (c) respectively. Results of numerical simulations (squares) for (a) an active fluid of spherical particles [3], (b) active polymer-like chains [4] and (c) sheared SGM system [5].

NSG is the incumbent of the Lee and William Abramowitz Professorial Chair of Biophysics. This research is made possible in part by the generosity of the Harold Perlman Family. SKN thanks the Koshland Foundation for funding through a fellowship.

Publisher's Note The EPJ Publishers remain neutral with regard to jurisdictional claims in published maps and institutional affiliations.

\section{References}

1. E. Ben-Isaac, É. Fodor, P. Visco, F. van Wijland, N.S. Gov, Phys. Rev. E 92, 012716 (2015).

2. S.K. Nandi, N. Gov, Eur. Phys. J. E 41, 117 (2018).

3. D. Loi, S. Mossa, L.F. Cugliandolo, Phys. Rev. E 77, 051111 (2008).

4. D. Loi, S. Mossa, L.F. Cugliandolo, Soft Matter 7, 3726 (2011).

5. L. Berthier, J.-L. Barrat, Phys. Rev. Lett. 89, 095702 (2002). 